\title{
Acute Lower Limb Ischemia as Presenting Symptom of Acute Myocardial Infarction Complicated With Left Ventricular Thrombus in a Young Male Patient: A Case Report
}

\author{
Aurora Bakallia, c, Krenare Limani ${ }^{a}$, Laura Leci-Tahirib, Sami Gjoka ${ }^{a}$, Edita Pllana ${ }^{\text {a }}$
}

\begin{abstract}
Left ventricular (LV) thrombus is one of myocardial infarction (MI) complications and if the thrombus is mobile and protruding, there is a high risk of embolization. It is exceptionally rare that the presenting serious symptom of acute MI is severe leg pain. We report here a rare case of a 36-year-old Caucasian male patient with unrecognized MI complicated by LV thrombus. His initial visit to the physician was due to sudden onset of severe left leg pain as a consequence of thromboembolism. Medical examination revealed occluded left femoral commune artery and femoral superficial artery, subacute MI and apical LV thrombus, which were treated accordingly. On follow-up, 2 months after hospital discharge, patient affirmed that he has been walking for approximately $3 \mathrm{~km}$ every day, without leg cramps or angina. Early recognition of MI, even when presented with atypical symptoms in young individuals and its prompt treatment is important to improve survival, as well as to reduce thrombotic events.
\end{abstract}

Keywords: Myocardial infarction; Acute limb ischemia; Symptom; Ventricular thrombus

\section{Introduction}

Left ventricular (LV) thrombus is a myocardial infarction (MI) complication [1]. Mobile and protruding thrombi are at higher risk of embolization [1].

We report here a rare case of a 36-year-old male patient with acute MI complicated by LV thrombus, whose initial visit to the physician was due to sudden onset of severe left leg pain

\footnotetext{
Manuscript accepted for publication April 07, 2016

${ }^{\mathrm{a} C l i n i c}$ of Cardiology, University Clinical Center of Kosova, Prishtine, Kosovo

${ }^{\mathrm{b}}$ Clinic of Vascular Surgery, University Clinical Center of Kosova, Prishtine, Kosovo

${ }^{\mathrm{c}}$ Corresponding Author: Aurora Bakalli, Clinic of Cardiology, University Clinical Center of Kosova, Prishtine, Kosovo. Email: abakalli@hotmail.com
}

doi: http://dx.doi.org/10.14740/jmc2480w as a consequence of thromboembolism. The value of this case presentation is that it is extremely rare that the presenting serious symptom of acute MI in a young male patient is its complication, namely thromboembolisation from the LV which was manifested by acute lower limb ischemia.

\section{Case Report}

A 36-year-old Caucasian male was transferred to the Cardiology Clinic from Vascular Surgery Clinic due to ECG changes consistent with MI. Four days earlier, patient was admitted to Vascular Surgery Clinic due to sudden severe pain of the left leg. Physical examination revealed that left leg was cold, whereas dorsalis pedis, posterior tibial, popliteal and femoral pulses were absent. There were no signs of chronic ischemic lower limb disease such as loss of leg hair, thinning of the skin or gangrenous changes. Doppler ultrasound showed occluded femoral commune artery and femoral superficial artery. Thrombectomy of the occluded arteries with Fogarty balloon catheter was performed urgently. Subsequent to the procedure, the pain disappeared, peripheral pulses were palpable and left leg was warm. He was transferred to Cardiology Clinic due to ECG changes that suggested subacute MI.

Regarding MI, patient referred that he did not have chest pain; however, he recalled that a few days prior to the leg pain, he had experienced upper abdominal pain and nausea but had not sought medical attention. Patient had negative family history for coronary artery disease. He was a smoker, and did not abuse narcotic substances. Patient denied other diseases. He was not aware earlier of diabetes, which was diagnosed for the first time during this hospitalisation.

Patient's BMI was $23.98 \mathrm{~kg} / \mathrm{m}^{2}$, belonging to normal weight category. Laboratory results showed initial glycemia of $19.36 \mathrm{mmol} / \mathrm{L}$, whereas cholesterol and triglycerides were $5.27 \mathrm{mmol} / \mathrm{L}$ and $1.77 \mathrm{mmol} / \mathrm{L}$, respectively. Troponine test was positive $(0.35 \mathrm{ng} / \mathrm{L})$ and lactate dehydrogenase level was elevated $(1,113 \mathrm{U} / \mathrm{L})$. Hemostasis tests and other laboratory tests were within normal reference range.

ECG showed sinus rhythm, heart rate of 76 beats per minute, Q waves with ST elevation in leads V1 to V3 and T wave inversion in leads D1, aVL, V4 to V6.

Echocardiography examination revealed akinetic LV apex 


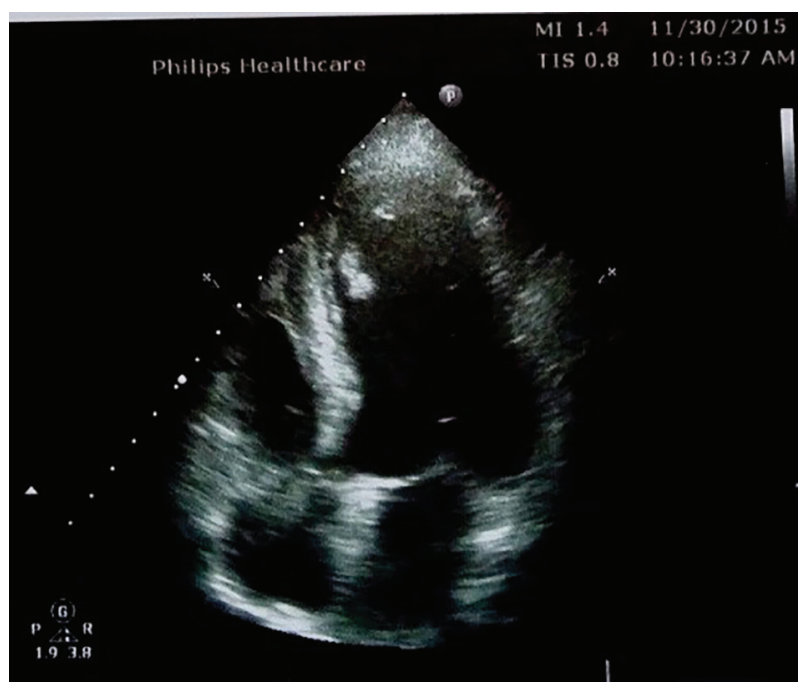

Figure 1. A protruding, well-contoured left ventricular thrombus on apical segment of the interventricular septum detected by echocardiography.

and a protruding, well-contoured, mobile thrombus, with a dimension of $12 \times 7 \mathrm{~mm}$ located in the apical segment of the interventricular septum (Fig. 1). LV ejection fraction was estimated $54 \%$.

Coronary angiography showed 95\% LAD stenosis of proximal segment, without atherosclerotic alterations in other coronary artery vessels (Fig. 2). Successful stenting of the LAD was achieved.

Funduscopic examination was completed to disclose whether patient had suffered from diabetes mellitus for longer period. Nevertheless, fundoscopy was unremarkable, thus excluding long standing diabetes mellitus.

Patient was treated with dual antiplatelet therapy, anticoagulant treatment, high-dose statin therapy, a beta blocker and

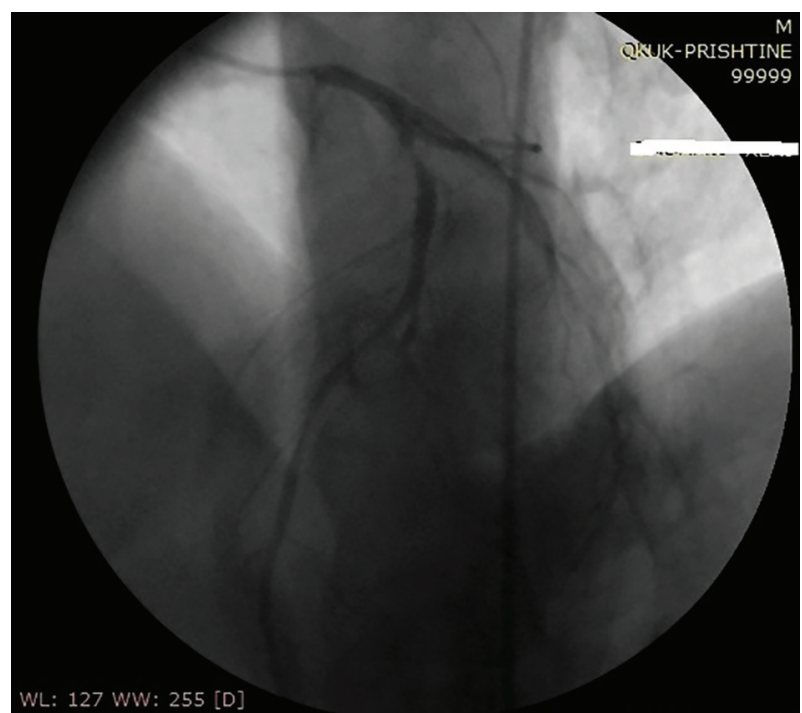

Figure 2. Coronary angiography showing 95\% LAD stenosis of the proximal segment.

oral antidiabetic medications.

Prior to hospital discharge, 2.5 weeks after hospitalisation in Cardiology Clinic, patient had no leg pain, but complained of numbness of the left foot. However, on physical examination, the left leg was warm; pulses on popliteal, dorsalis pedis and posterior tibial artery were absent; no motor deficit of the limb was detected. We performed angiography of the lower limbs, which illustrated occlusion of the left popliteal artery at the level of the second segment with feeble collaterals for distal arteries. There were no atherosclerotic changes evident on other arteries of the lower limbs (Fig. 3). Control echocardiography showed that LV thrombus had dissolved.

On follow-up, 2 months after hospital discharge, patient was feeling well. He affirmed that he has been walking for

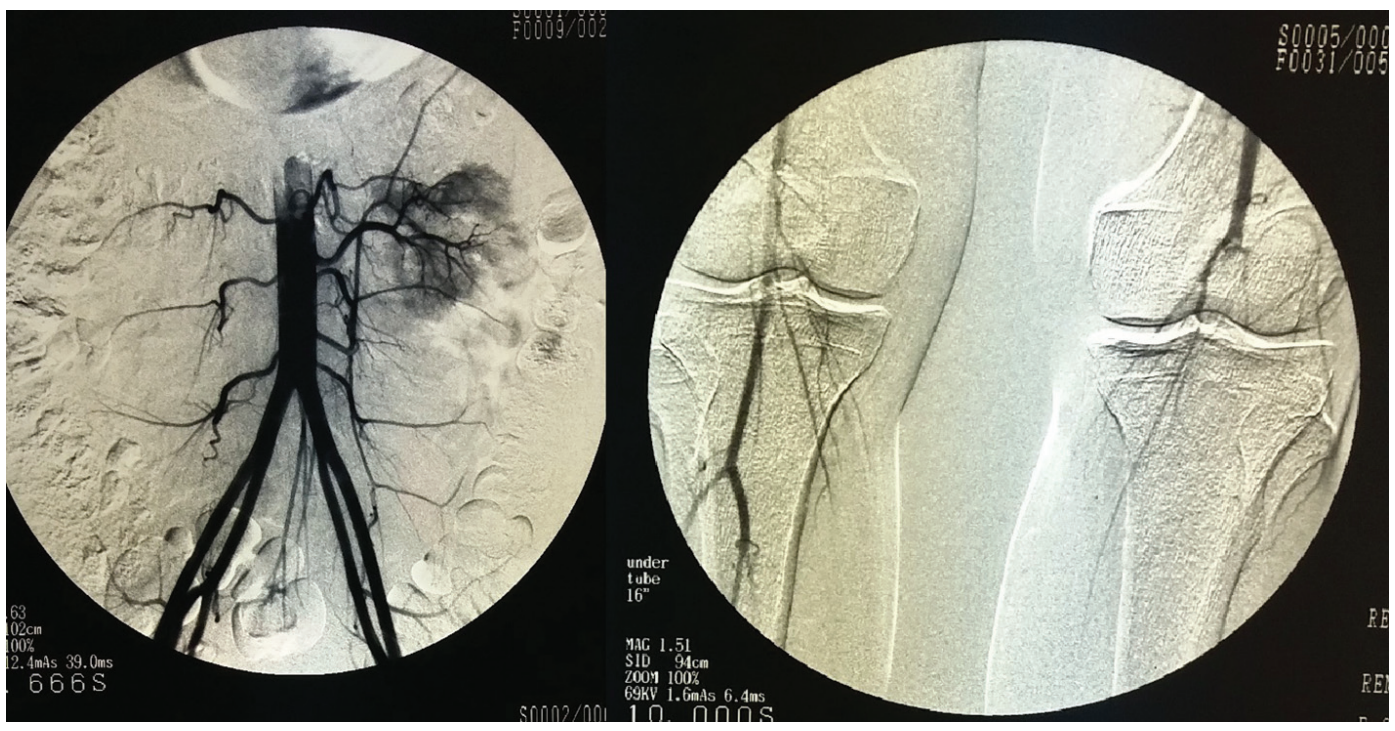

Figure 3. Peripheral angiography of the lower limbs showing normal femoral arteries (left image) and occluded left popliteal artery (right image). 
approximately $3 \mathrm{~km}$ every day, without leg cramps or angina.

\section{Discussion}

The common symptom of chest pain is not pathognomonic for MI, as patients with acute MI may have atypical presentations such as dyspnea, abdominal or epigastric pain, nausea, vomiting, syncope, etc. $[2,3]$. It is not rare that the diagnosis of MI is missed due to patient's atypical symptoms. This brings up the importance of awareness for the society at large, on different forms of clinical presentation of acute MI, and importance of recognizing symptoms in early diagnosis and long-term outcomes. Treatment of MI by early thrombolysis or better yet by percutaneous coronary intervention (PCI) has led to reduction of LV thrombus formation [4]. Therefore, an untreated MI has a higher likelihood for development of LV thrombus. Certain thrombi features, such as mobility and protrusion, as mentioned earlier, make them more hazardous for embolization [1].

The exact cause of MI in our young patient is not clear, although it is likely that his diabetes and smoking contributed to accelerated atherosclerosis of coronary arteries.

Lower limb ischemia in this patient, on the other hand, is believed to be as a consequence of thromboembolus, most likely arriving from the LV, as we found a protruding, mobile thrombus in the LV apex. No atherosclerosis detected on peripheral angiography, as well as absence of signs of chronic ischemic disease on the leg favor our assumption. Fortunately, the leg was saved, although the popliteal artery remained occluded. We believe that this was due to Fogarty thrombectomy complication, partial thrombus drive to popliteal artery from the femoral artery or endothelial damage that could cause thrombosis. Pushing thrombus to distal vessels is a rare but recognized complication of endovascular thrombectomy procedures [5]. As the vascular surgeons report that all the peripheral pulses were palpable following the thrombectomy procedure, a more likely explanation of popliteal artery occlusion seen on peripheral angiography performed 3 weeks after the procedure is thrombosis due to endothelial damage. Nevertheless, there are cases in the medical literature with worse outcomes, such as that of an obese 35-year-old male patient with MI, LV thrombus and multiple thromboemboli that ended up with bilateral above knee amputations [6].

\section{Conclusion}

Early recognition of MI, even when presented with atypical symptoms in young individuals and its prompt treatment is important to improve survival, as well as to reduce thrombotic events. Patients should also be aware of the treatment options and the rewards of early MI management. Fogarty balloon catheter thrombectomy may be complicated by distal thrombus formation.

\section{Acknowledgement}

We would like to thank all the medical personnel of Cardiology and Vascular Surgery Departments that were involved in diagnosing and treating this patient.

\section{Competing Interests}

The authors declare that they have no competing interests.

\section{Author Contributions}

$\mathrm{AB}$ and LLT examined the patient, analyzed and interpreted patient data. AB was a major contributor in writing the manuscript, also. SG performed, analyzed and interpreted the coronary angiography and stenting. KL and EP contributed on collecting the patient data as well as relevant papers regarding this case. All authors read and approved the final manuscript.

\section{References}

1. Meltzer RS, Visser CA, Fuster V. Intracardiac thrombi and systemic embolization. Ann Intern Med. 1986;104(5):689-698.

2. Sigurdsson E, Thorgeirsson G, Sigvaldason H, Sigfusson N. Unrecognized myocardial infarction: epidemiology, clinical characteristics, and the prognostic role of angina pectoris. The Reykjavik Study. Ann Intern Med. 1995;122(2):96-102.

3. Canto JG, Shlipak MG, Rogers WJ, Malmgren JA, Frederick PD, Lambrew CT, Ornato JP, et al. Prevalence, clinical characteristics, and mortality among patients with myocardial infarction presenting without chest pain. JAMA. 2000;283(24):3223-3229.

4. Delewi R, Zijlstra F, Piek JJ. Left ventricular thrombus formation after acute myocardial infarction. Heart. 2012;98(23):1743-1749.

5. Yavuz C, Cil H, Demirtas S, Yolbas I, Benli E. Successful embolectomy in a femoral artery thrombosis caused by femoral artery catheterization in a infant. Eur Rev Med Pharmacol Sci. 2012;16(Suppl 4):32-34.

6. Chetwood A, Sanders A, Saweirs M, Thapar A, Davies AH. Multiple arterial emboli secondary to left ventricular thrombus in a 35 -year-old obese male. J Cardiovasc Dis Res. 2010;1(4):203-205. 